\title{
An LED Drive Circuit with Constant-Output- Current Control and Constant-Luminance Control
}

\author{
Masahiro Nishikawa $^{\dagger}$, Yoichi Ishizuka ${ }^{\dagger}$, Hirofumi Matsuo ${ }^{\dagger}$ and Koichi Shigematsu ${ }^{\dagger \dagger}$ \\ Nagasaki University ${ }^{\dagger}$, Nagasaki, Japan Ansoft Japan ${ }^{\dagger \dagger}$, Kanagawa, Japan
}

\begin{abstract}
A Single LED is very small, and their luminance is related to the amount of driving current. Sufficient luminance for an illuminator requires many LEDs to be connected together and a constant current supplied to each LED. Therefore, the LED drive circuit must have constant-output-current control and/or constant-luminance control. We propose an LED drive circuit that consists of a boost-type DC-DC converter with constant-output-current control and constant-luminance control. This circuit is a switching regulator-type circuit controlled by pulse width modulation (PWM). Both the power and the constant-current control can be provided by a single system, without any ballast resistor or auxiliary current control circuit.
\end{abstract}

\section{INTRODUCTION}

The light emitting diode (LED) has been drawing attention as a state-of-the-art illuminator. Advancements in brightLED-fabrication processes, device designs and assembly technologies have led to an increase in the performance of LEDs and a reduction in cost.

Single LEDs are very small, and their luminance is related to the amount of driving current. To obtain sufficient luminance for an illuminator, a number of LEDs must be connected together and a constant current must be supplied to each of the LEDs for equivalent luminance. A serial connection is superior to a parallel connection, because a constant current can be simply supplied to each LED belonging to an array. However, the total voltage and total current of a serial connection varies, depending on the occurrence of shortcircuit LED breakdowns. Consequently, the LED drive circuit must have constant-output-current control and/or constant-luminance control.

An LED drive circuit is proposed, which consists of a boost-type DC-DC converter with constant-output-current control and constant-luminance control. This circuit is a switching regulator-type circuit controlled by pulse width modulation (PWM). Both the power and the constant-current control can be provided by a single system, without any ballast resistor or auxiliary current control circuit. Constant luminance control can be provided using photodiodes.

In section II, the electrical properties and the type of connection for the LEDs are described. In section III, the proposed driving circuit method is introduced. Section IV provides the experimental and simulation results.

\section{The Electrical Properties of THE LED}

A. The Electrical Properties of the LEDs

Fig. 1 shows the forward current and luminance versus the forward voltage characteristics of the LED (NSPW500BS) used in these experiments. The forward current begins to flow at a forward voltage of $2.6 \mathrm{~V}$. The forward current increases with an increase in the forward voltage within the range of the maximum ratings for the forward current. Luminance also increases in proportion to an increase of the forward current.

\section{B. Type of Connection for the LEDs}

Generally, a single LED is very small. To obtain sufficient luminance for an illuminator, many LEDs must be connected together, and a constant current supplied to each LED. There are two types of connection, n-paralleled connection and series connection, as shown in Figs. 2 and 3, respectively.

In Fig. 2, $\mathrm{R}_{\mathrm{pk}}$ is the ballast resistance, and $\mathrm{I}_{\mathrm{Fpk}}$ is the drive current of LED $\mathrm{k}$ and is represented as

$$
I_{F p k}=\frac{E_{i}-V_{F k}}{R_{b p k}} \text {. }
$$

From (1), it can be seen that each $\mathrm{I}_{\mathrm{F}}$ cannot have an equivalent value when each $R_{b p k}$ has a deviation. In addition, the value of the ballast resistance is increased in proportion to the number of LEDs.

In Fig. $3, \mathrm{E}_{\mathrm{i}}$ is the input voltage, $\mathrm{E}_{\mathrm{o}}$ is the output voltage, $D_{1}$ to $D_{n}$ represent the single LEDs, $I_{F}$ is the drive current, $V_{F n}$ is the forward voltage of the LEDs and $R_{b s}$ is the ballast resistance. $\mathrm{I}_{\mathrm{F}}$ is represented as

$$
I_{F}=\frac{E_{i}-\sum_{k=1}^{n} V_{F k}}{R_{b s}} .
$$

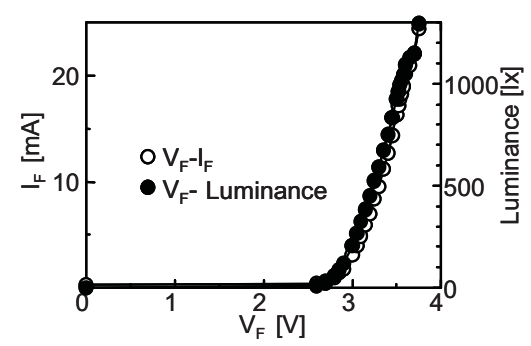

Fig. 1. $\mathrm{I}_{\mathrm{F}}$ and $\mathrm{F}$ versus $\mathrm{V}_{\mathrm{F}}$.(White LED: NSPW500BS) 
From (2), it can be seen that the drive current $I_{F}$ must be an equivalent value even if $\mathrm{E}_{\mathrm{i}}, \mathrm{V}_{\mathrm{Fk}}$ and $\mathrm{R}_{\mathrm{bs}}$ have some deviation. Therefore, a series connection is more effective as the drive circuit for lighting, because of the stability of the brightness for each LED.

However, the total voltage and total current of a serial connection varies depending on the occurrence of short-circuit breakdown of LEDs. Such an occurrence affects the change of luminance of the LEDs. In a worst case scenario, the LEDs will continuously breakdown.

Considering this, an active constant current compensation circuit is definite requirement for a series connection driving circuit.

\section{Relation of Luminous Efficiency and Temperature}

One of the advantages of LEDs is long life superiority over fluorescent and incandescent lamps. However, the luminous efficiency is decreased by device deterioration due to ageing. The luminous efficiency is also changed by internal and external temperatures. Although the luminance of LEDs is related to the amount of driving current, the luminance is also changed by the environment. To confirm this problem, constant temperature experiments were performed.

10 LEDs were connected in series with ballast resistance; the same configuration as shown in Fig. 3. Figs. 4 and 5 show the variation in the voltage of the ballast resistance $V_{R s}$ $=10 \Omega$, the output voltage $\mathrm{E}_{\mathrm{o}}$, drive current $\mathrm{I}_{\mathrm{F}}$ and the luminance of the LEDs as a function of operation time.

The results indicate that $\mathrm{E}_{\mathrm{o}}$ is decreased by $0.06 \mathrm{~V}$ after ten minutes has passed. The average error for $\mathrm{E}_{\mathrm{o}}$ was $0.17 \%$.

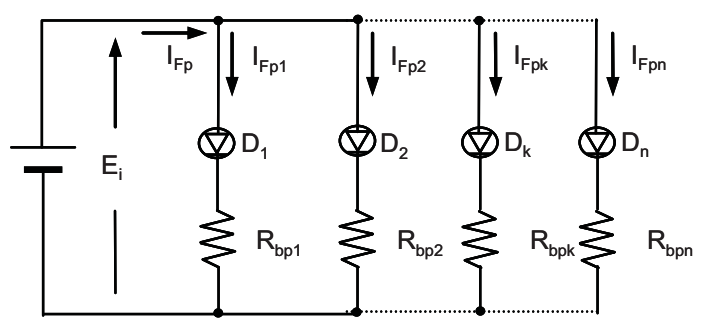

Fig. 2. A parallel connection.

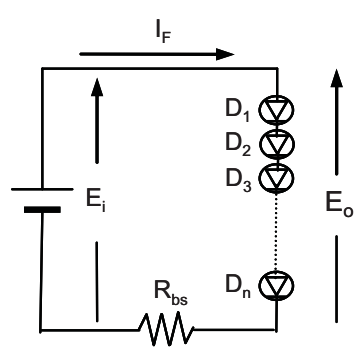

Fig. 3. A series connection.

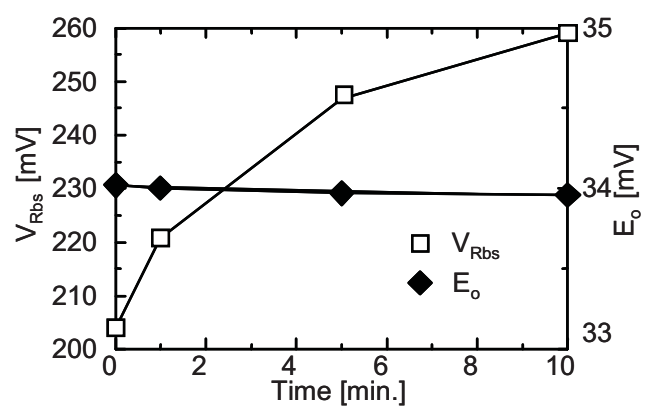

Fig. 4. Time versus $\mathrm{I}_{\mathrm{F}}$ and $\mathrm{E}_{\mathrm{o}}$.

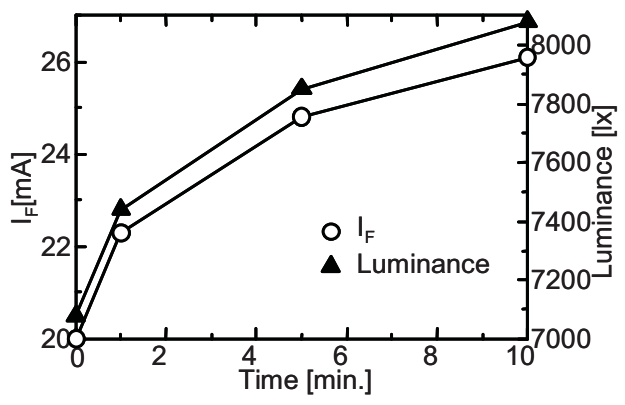

Fig. 5. Time versus $\mathrm{I}_{\mathrm{F}}$ and Luminance.

$\mathrm{I}_{\mathrm{F}}$, with an initial value of $20 \mathrm{~mA}$, became $26.01 \mathrm{~mA}$ after ten minutes. The luminance, which was initially 7080 lx, became $8080 \mathrm{~lx}$ after ten minutes. The luminance increase of approximately $1000 \mathrm{~lx}$ is proportional to the increase of $\mathrm{I}_{\mathrm{F}}$. Therefore, it is apparent that the forward voltage $V_{F}$ is changed by the internal temperature of LEDs with a continuous driving current. The overcurrent of $I_{F}$ occurs with a decrease in $\mathrm{V}_{\mathrm{F}}$. In addition, $\mathrm{V}_{\mathrm{Rs}}$, which was initially $204 \mathrm{mV}$, became $259 \mathrm{mV}$ after ten minutes, an increase of approximately $55 \mathrm{mV}$. Therefore, it is seen that the ballast resistance does not suppress a decrease of $\mathrm{V}_{\mathrm{F}}$ caused by the LEDs internal temperature change. Consequently, a current compensation circuit is effective for constant current flow, regardless of a decrease in $\mathrm{V}_{\mathrm{F}}$ from internal temperature change or short circuiting of LEDs.

\section{Proposed Driving Circuit Method \\ A. Circuit Conditions}

We propose an LED drive circuit that consists of a boosttype DC-DC converter with constant-output-current control and constant-luminance control as a drive circuit for lighting that uses LEDs with the features shown in section II. With this drive circuit, a system becomes small and lightweight, and has high efficiency. Current compensation is also realized. In this section, the composition and operation of a boost-type DC-DC converter with constant-output-current control and the constant-luminance control circuit are given. 
Fig. 6 shows a boost-type DC-DC converter with constantoutput-current control, where $\mathrm{S}$ is the switch, $\mathrm{D}$ is the diode, $\mathrm{L}$ is the inductor, $\mathrm{C}$ is the output capacitor, $\mathrm{D}_{1}$ to $\mathrm{D}_{\mathrm{n}}$ are $\mathrm{n}$ single white LEDs, $R_{d}$ is the current detection resistance, $R_{1}$ and $R_{2}$ is the resistance that divides the output voltage $E_{o}, I_{F}$ is the drive current, $\mathrm{V}_{\mathrm{Fn}}$ is the forward voltage of the LEDs and $E_{o}$ is the sum of the voltage of $n$ single LEDs. The input of the constant-output-current control circuit is the difference voltage $e_{s}$, between $R_{d}$ and $R_{2}$.

In this study, the following assumptions were determined for the analysis.

(1) L and C are assumed to be sufficiently large. The DC power voltage ripple of the voltage and the current is disregarded.

(2) The forward voltage and forward resistance of diode D is assumed to be 0 , and the backward resistance is assumed to be infinite. $\mathrm{D}$ is turned on by a positive voltage and turned off by zero or negative voltage. The recovery time is assumed to be zero.

(3)Parasitic resistance and capacitance of transistor S is assumed to be zero. The turn-on voltage and resistance is assumed to be zero. The turn-off resistance is assumed to be infinite. In addition, the turn-on time and the turn-off time are disregarded, assuming that they are very small compared with the on-term and the off-term period.

(4) The relation of internal loss r, and inductance L, is determined by the following conditions, where $\mathrm{f}_{\mathrm{s}}$ is the switching frequency.

$$
f_{s} L \geq r \text {. }
$$

\section{B. Constant-output-current control of a boost-type DC-DC converter}

In the drive circuit used in this research, output-current is detected by the resistance $R_{d}$. The equation is derived with respect to the constant-output-current control coefficient.

$E_{s}$ is the mean value of $e_{s}$, and is represented as follows:

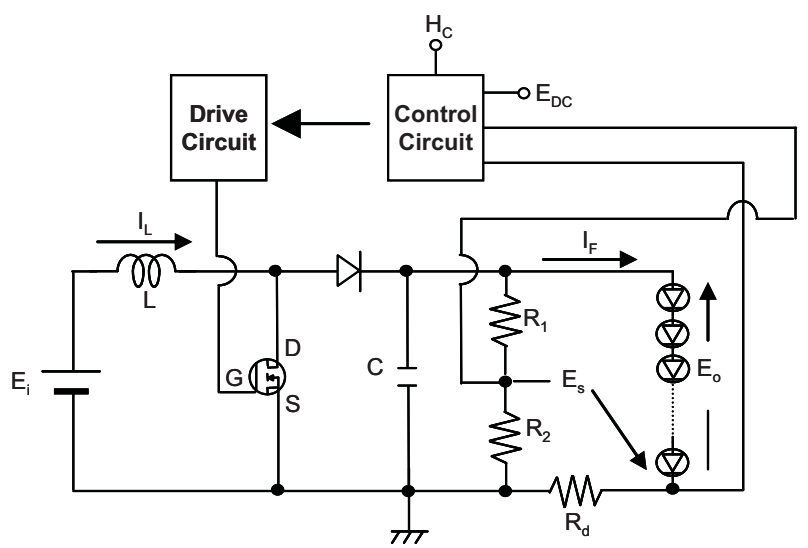

Fig. 6. The proposed boost-type DC-DC converter with constant-outputcurrent control.

$$
E_{s}=V_{R d}-V_{R 2}
$$

where $V_{R d}$ and $V_{R 2}$ are the voltages of $R_{d}$ and $R_{2}$.

\section{The Constant-Output-Current Control Coefficient and Output Characteristics}

Fig. 7 shows the proposed constant-output-current control circuit.

In this circuit, the duty ratio $\mathrm{D}$, is represented by the following equation.

$$
D=\frac{V_{s}}{E_{D C}}=\frac{H_{C} \cdot E_{s}+E_{B}}{E_{D C}}
$$

where $\mathrm{E}_{\mathrm{DC}}$ is the reference voltage and $\mathrm{H}_{\mathrm{C}}$ is a coefficient. $\mathrm{V}_{\mathrm{s}}$ has a saw-tooth-like amplitude, and is represented as follows:

$$
V_{S}=\left(\frac{R_{l 1}}{R_{l 2}}\right) \cdot E_{S}+E_{B}
$$

$E_{B}$ is the bias voltage and determines the ratings value of $I_{F}$, and is represented as follows

$E_{B}=I_{F} \cdot\left(\frac{R_{l 2}}{R_{l l}}\right) \cdot\left(\frac{R_{1} \cdot R_{S}}{R_{1}+R_{2}}+\frac{R_{S}+r}{H_{C} \cdot E_{i}}\right)-E_{o} \cdot\left(\frac{R_{l 2}}{R_{l l}}\right) \cdot\left(\frac{R_{2}}{R_{l}+R_{2} \cdot R_{d}}-\frac{1}{E_{i} \cdot H_{C}}\right)$

In OP1, the constant current signal $\mathrm{E}_{\mathrm{s}}$, is detected and the signal $E_{1}$ is output.

$E_{1}$ is represented by the following equation.

$$
E_{1}=\left(\frac{R_{l 2}}{R_{l 1}}\right) \cdot E_{s}
$$

where $R_{11} / R_{12}$ is the direct-current gain in the constant-current control circuit.

In OP2, $E_{1}$ is adjusted in the bias with $E_{B}$ and the resulting $E_{2}$ is output.

For a saw-tooth oscillation circuit, a saw-tooth wave of amplitude $\mathrm{V}_{\mathrm{s}}$ is output.

For COMP1, the pulse width is determined by comparing $\mathrm{V}_{\mathrm{s}}$ with $\mathrm{E}_{\mathrm{DC}}$.

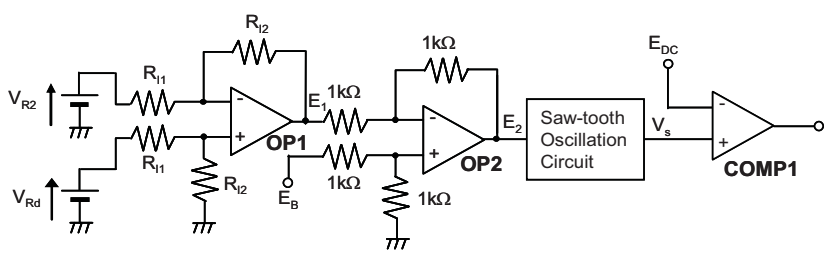

Fig. 7. The proposed constant-output current control circuit. 
$\mathrm{H}_{\mathrm{C}}$ is represented by the following control equation.

$$
H_{C}=\frac{R_{l 1} / R_{l 2}}{E_{D C}} .
$$

From (5) and (9), it is apparent that a constant current is supplied to the LED by pulse width modulation (PWM).

$\mathrm{E}_{0}^{*}$ is the ratings output voltage of a boost-type DC-DC converter and is represented by the following equation.

$$
1-\frac{I_{F}}{I_{F}{ }^{*}}=\frac{G-1}{r+R_{S} \cdot\left(1+E_{i} \cdot H_{C}-G\right)} \cdot \frac{E_{o}{ }^{*}-E_{o}}{I_{F}{ }^{*}}
$$

where $\mathrm{G}$ is the constant-output-current control coefficient, and is represented as follows:

$$
G=E_{i} \cdot H_{C} \cdot \frac{R_{2}}{R_{1}+R_{2}} .
$$

In (9), the relation between $\mathrm{I}_{\mathrm{F}}{ }^{*}$ and $\mathrm{I}_{\mathrm{SC}}$ is represented by the following equation.

$$
1-\frac{I_{S C}}{I_{F}{ }^{*}}=\frac{G-1}{r+R_{S} \cdot\left(1+E_{i} \cdot H_{C}-G\right)} \cdot \frac{E_{o}{ }^{*}}{I_{F}{ }^{*}}
$$

where $\mathrm{I}_{\mathrm{F}}{ }^{*}$ is the ratings output current of a boost-type DC-DC converter and $I_{\mathrm{SC}}$ is the short-circuit current when $\mathrm{E}_{\mathrm{o}}=0$ for $\mathrm{I}_{\mathrm{F}}$.

In (12), the right hand side of the equation becomes 0 at $\mathrm{G}$ $=1$. Therefore, $\mathrm{I}_{\mathrm{F}}^{*}=\mathrm{I}_{\mathrm{SC}}$ and this is assumed to be a constantoutput-current characteristic. The right hand side of (12) becomes positive at $\mathrm{G}>1$, and $\mathrm{I}_{\mathrm{F}}{ }^{*}<\mathrm{I}_{\mathrm{SC}}$ with this assumed to be a fall-back characteristic. The right hand side of (12) becomes positive at $\mathrm{G}<1$, with $\mathrm{I}_{\mathrm{F}}{ }^{*}>\mathrm{I}_{\mathrm{SC}}$, and is assumed to be a drooping characteristic. Therefore, controlling the inclination of the current becomes possible by causing $\mathrm{G}$ to change, where $\mathrm{G}$ is defined as the constant-output current control coefficient.

\section{Constant-Luminance Control of a Boost-Type DC-DC Converter}

The luminance of LEDs is related to the amount of driving current. Therefore, stabilization of luminance can be performed by controlling the amount of flow of driving current. In section III $C$, it was shown that control of the driving current by causing $\mathrm{G}$ to change can stabilize the luminance.

Fig. 8 shows a constant-luminance control circuit. In this study, constant-luminance control using the current control method with a photodiode, which is a semiconductor element that converts light into current, is examined.

In this method, the luminance of LEDs is converted into current and detected as a voltage $E_{p 1}$. The value of $E_{p l}$ is amplified by an error amplifier and is fed back to the voltage $E_{D C}$ of the constant-output-current control circuit. At OP3, the voltage $E_{p 1}$ is reversed to $E_{p 2}$. At OP4, the difference between

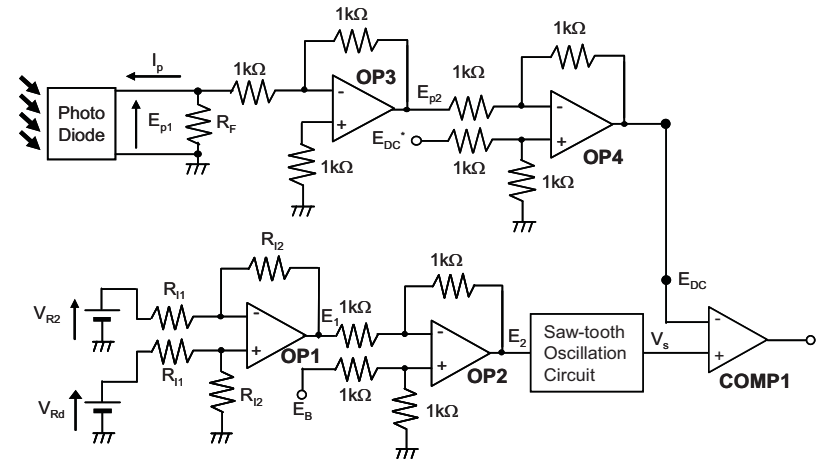

Fig. 8. The proposed constant-luminance control circuit.

$\mathrm{E}_{\mathrm{p} 2}$ and $\mathrm{E}_{\mathrm{DC}}{ }^{*}$ is amplified to $\mathrm{E}_{\mathrm{DC}}, \mathrm{E}_{\mathrm{DC}}$ is represented by the following equation:

$$
\begin{aligned}
E_{D C} & =E_{D C}{ }^{*}-E_{p 2} \\
& =E_{D C}{ }^{*}-\left\{-\left(-R_{F} \cdot I_{p}\right)\right\} \\
& =E_{D C}{ }^{*}-R_{F} \cdot I_{p}
\end{aligned}
$$

where $I_{p}$ is the output current of the photodiode.

The duty ratio $\mathrm{D}$, is represented by the following equation.

$$
D=\frac{V_{s}}{E_{D C}}=\frac{H_{C} \cdot E_{s}+E_{B}}{E_{D C}{ }^{*}-R_{F} \cdot I_{p}}
$$

In (14), the pulse width is determined by comparison of $E_{D C}$ with $\mathrm{V}_{\mathrm{s}}$, and is controlled by changing the $\mathrm{E}_{\mathrm{DC}}$ of the comparator.

Therefore, luminance stabilization for a changing number of LEDs and a change in the $\mathrm{V}_{\mathrm{F}}$ caused by temperature change can be realized.

\section{EXPERIMENTAL AND SIMULATION RESULTS}

In this section, the control scheme is described precisely and simulation with Ansoft Simplorer and experimental results are used to clarify the validity of the drive circuit. The maximum number of LED is assumed to be 10, and Table 1 shows the parameter values at $\mathrm{G}=1 . \mathrm{E}_{\mathrm{B}}$ is determined with disregard for $r$ given in (7).

Fig. 9 shows $I_{F}$ versus the number of LEDs. For the experimental and the simulated results, a constant current is achieved at a rated current of $20 \mathrm{~mA}$ without dependence on the number of LEDs between 5 and 10 . The average error is $0.2 \%$, and the maximum error was $1 \%$ for an array of 8 LEDs. Fig. 10 shows power efficiency $\eta$, versus the number of LEDs. The average power efficiency is over $90 \%$, and the maximum power efficiency is $93.4 \%$ for an array of 5 LEDs.

Fig. 11 shows the drive current, $\mathrm{I}_{\mathrm{F}}$, versus the number of LEDs in constant-current control mode and constant-luminance mode. Fig. 12 shows the luminance versus the number of LEDs in constant-current control mode and constant-luminance mode. Considering both Figs. 11 and 12 together suggests that constant-luminance control allows adjustment of the luminance by linear control of $\mathrm{I}_{\mathrm{F}}$. The average error of luminance was within $1.5 \%$ and the maximum error was $3 \%$ for an array of 9 LEDs. 


\section{CONCLUSION}

An LED drive circuit that consists of a boost-type DC-DC converter with constant-output-current control and constantluminance control is proposed. The results are concluded as follows:

(1) A switching power supply method is used, resulting in low power consumption and high efficiency.

(2) The ability to supply a constant current to each LED, regardless of the number of changes of LEDs at $\mathrm{G}=1$ was confirmed by both experiment and simulation.

(3) Luminance can be constantly maintained for a photodiode by current control, and the change in luminance controlled under certain conditions.

In addition, the proposed circuit provides high power efficiency.

The luminous efficiency of LEDs is affected by the temperature. Therefore, future research will focus on the development of a drive circuit for high luminance efficiency and avoidance of internal temperature rises. In addition, digital control will be examined.

Table I

SIMULATION AND EXPERIMENTAL CONDITIONS.

\begin{tabular}{|c||c|c|}
\hline & Simulation & Experiment \\
\hline $\mathrm{E}_{\mathrm{i}}[\mathrm{V}]$ & 12 & 12 \\
\hline $\mathrm{E}_{\mathrm{o}}{ }^{*}[\mathrm{~V}]$ & 36 & 36 \\
\hline $\mathrm{I}_{\mathrm{F}}{ }^{*}[\mathrm{~mA}]$ & 20 & 20 \\
\hline $\mathrm{f}_{s}[\mathrm{kHz}]$ & 100 & 100 \\
\hline $\mathrm{R}_{\mathrm{d}}[\Omega]$ & 10 & 10 \\
\hline $\mathrm{L}[\mathrm{mH}]$ & 1 & 1 \\
\hline $\mathrm{C}[\mu \mathrm{F}]$ & 5 & 5 \\
\hline $\mathrm{G}$ & 1 & 1 \\
\hline $\mathrm{R}_{\mathrm{t}}[\mathrm{k} \Omega]$ & 119 & 119 \\
\hline $\mathrm{R}_{2}[\mathrm{k} \Omega]$ & 1 & 1 \\
\hline $\mathrm{R}_{\mathrm{II}}[\mathrm{k} \Omega]$ & 10 & 10 \\
\hline $\mathrm{R}_{\mathrm{I}}[\mathrm{k} \Omega]$ & 100 & 100 \\
\hline $\mathrm{E}_{\mathrm{B}}[\mathrm{V}]$ & 2 & 1.8 \\
\hline $\mathrm{E}_{\mathrm{DC}}[\mathrm{V}]$ & 1 & 1 \\
\hline $\mathrm{E}_{\mathrm{DC}}{ }^{*}[\mathrm{~V}]$ & - & 1 \\
\hline $\mathrm{R}_{\mathrm{F}}[\Omega]$ & - & 100 \\
\hline
\end{tabular}

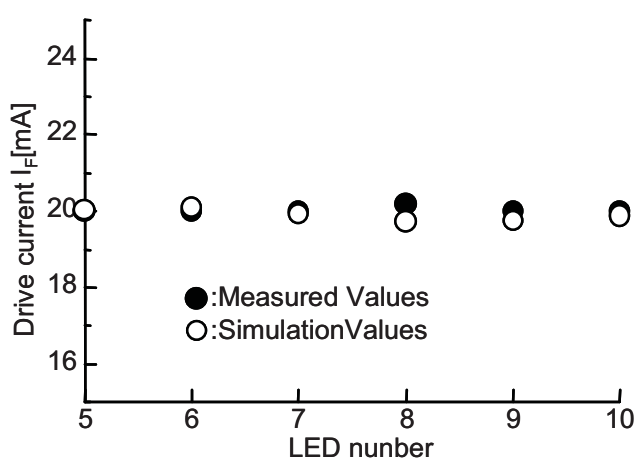

Fig. 9. $\mathrm{I}_{\mathrm{F}}$ versus the number of LEDs.

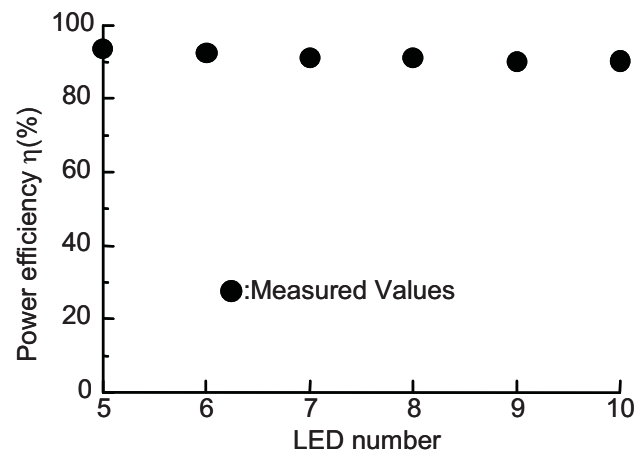

Fig. 10. Power efficiency, $\eta$, versus the number of LEDs

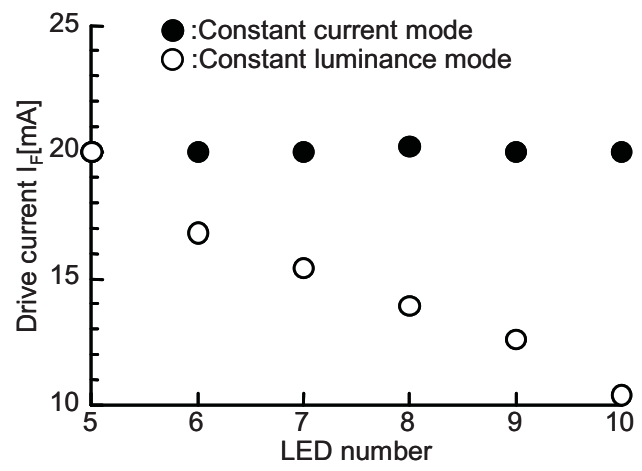

Fig. 11. $\mathrm{I}_{\mathrm{F}}$ versus the number of LEDs.

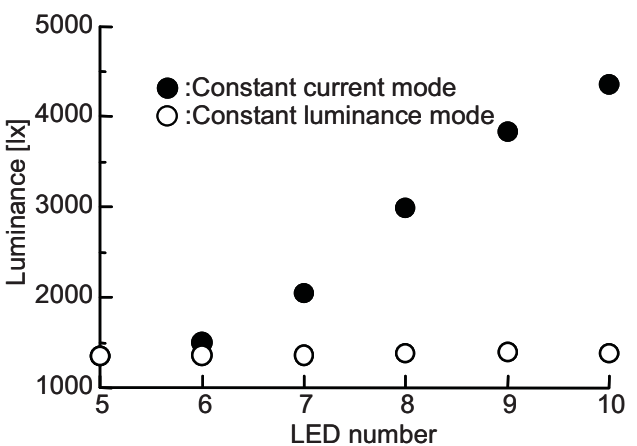

Fig. 12. Luminance versus the number of LEDs. 
ACKNOWLEDGEMENT

This work was supported in part by the Grant-in-Aid for Scientific Research (No.16560246) of Japan Society for the Promotion of Science and the Ministry of Education, Science, Sports and Culture.

\section{REFERENCES}

[1] K. Morihori, Y. Ishizuka, H. Matsuo, "Consideration of A Drive Circuit for LED with Constant-Current Control”, IEICE Technical Report EE 2004-68(2005-02), pp.13-19, February, 2005.

[2] M. Nishikawa, K. Morihori, Y. Ishizuka, H. Matsuo, "Static Characteristics of A Drive Circuit for LED with Constant-Current Control", Annual Conference of IEI-J, pp.251, July, 2005.

[3] M. Nishikawa, Y. Ishizuka, H. Matsuo, K. Morihori: "Static Characteristics of A Drive Circuit for LED with Constant-Current Control and Constant-luminance Control”, IEICE Technical Report EE2005-72(2006-02), pp.67-72, September, 2005.

[4] C.-C.Chen , C.-Y. Wu and T.-F. Wu, "LED Back-Light Driving System for LCD Panels", IEEE APEC 2006, pp. 381-385, March.19, 2006.

[5] O. Ronat, P. Green, S. Ragona, "Accurate current control to drive high power LED strings”, IEEE APEC 2006, pp. 376-380, Malch.19, 2006.

[6] S.Muthu, F. J. Schuurmans and M. D. Pashley, "Red, Green, and Blue LED Based White Light Generation Issues and Control", in Proc. Industry Applications Conf., pp.327-333, 2002. 